\title{
Platelet indices in patients with infective endocarditis and embolic events; confounding factors should be considered
}

I read the article published by Ileri et al. [1] with a great interest. They compared mean platelet volume (MPV) and platelet distribution width (PDW) in infective endocarditis (IE) patients who developed embolic events, those who did not, and controls. MPV at hospital admission was higher in patients who had embolic events in the follow-up period compared to both those who did not have embolic events and control subjects. Similarly, patients with embolic events had increased PDW compared to non-embolic ones and control group. I would like to congratulate the authors on this well designed and written study. On the other hand, I want to mention minor criticism on this study from methodological aspect.

In general, the method of MPV measurement is correct. However, it has to be kept in mind that there are significant associations of MPV with obesity, hypertension, smoking, type 2 diabetes mellitus, pre-diabetes, hypercholesterolemia, coronary heart disease, metabolic syndrome, statin and some antihypertensive use, and atrial fibrillation [2-4]. The authors excluded patients with prosthetic heart valves, patients using antiplatelet drugs, and patients with hematological disorders or cancer. However, they did not mention the body mass index, blood pressure levels, smoking status, and levels of glucose and lipids in patients with IE and embolic event, in patients with IE and no embolic event and control subjects. It has been demonstrated that obesity, hypertension, smoking, type 2 diabetes mellitus, pre-diabetes, and hypercholesterolemia increase MPV [2-4].

In their study, the authors found no significant difference between patients with IE who had no embolic event and control subjects. As a difference from this result, previous studies demonstrated higher MPV in patients with IE who had no embolic event to this in control subjects [5, 6]. We believe that if the authors had considered the above-mentioned confounding factors, they would perhaps have found different results. Moreover, previous studies demonstrated that MPV was significantly elevated in patients with aortic and mitral stenosis compared to control subjects, possibly due to shear stress induced platelet activation [7, 8]. However, the authors did not also mention the basal valvular status of the patients. Presence of basal mitral and/ /or aortic stenosis by itself can cause elevated MPV.

Recently, MPV has gained great attention especially in cardiovascular disease in diagnostic and prognostic aspect. In comparison to smaller ones, larger platelets have more granules, aggregate more rapidly with collagen, have higher thromboxane A2 level and express more glycoprotein $\mathrm{Ib}$ and IIb/IIIa receptors [2]. We believe that MPV can be affected by many cardiovascular risk factors. We believe that, it would have been useful if the authors had provided this information.

Conflict of interest: None declared

\section{References}

1. Ileri M, Kanat S, Orhan G et al. Increased mean platelet volume in patients with infective endocarditis and embolic events. Cardiol J, 2014 Mar 27. doi: 10.5603/CJ.a2014.0021 [Epub ahead of print].

2. Vizioli L, Muscari S, Muscari A. The relationship of mean platelet volume with the risk and prognosis of cardiovascular diseases. Int J Clin Pract, 2009; 63: 1509-1515.

3. Varol E, Akcay S, Icli A et al. Mean platelet volume in patients with prehypertension and hypertension. Clin Hemorheol Microcirc, 2010; 45: 67-72.

4. Varol E, Icli A, Kocyigit S, Erdogan D, Ozaydin M, Dogan A. Effect of smoking cessation on mean platelet volume. Clin Appl Thromb Hemost, 2013; 19: 315-319.

5. Cho SY, Jeon YL, Kim W et al. Mean platelet volume and mean platelet volume/platelet count ratio in infective endocarditis. Platelets, 2013 Nov 8 [Epub ahead of print].

6. Icli A, Tayyar S, Varol E et al. Mean platelet volume is increased in infective endocarditis and decreases after treatment. Med Princ Pract, 2013; 22: 270-273.

7. Varol E, Ozaydin M, Türker Y, Alaca S. Mean platelet volume, an indicator of platelet activation, is increased in patients with mitral stenosis and sinus rhythm. Scand J Clin Lab Invest, 2009; 69: 708-712.

8. Varol E, Arslan A, Yucel H, Ozaydin M, Erdogan D, Dogan A. Increased mean platelet volume in patients with aortic stenosis. Clin Appl Thromb Hemost, 2011; 17: E17-E20.

Ercan Varol, $M D$

Department of Cardiology, Faculty of Medicine,

Suleyman Demirel Univesitesi Tip Fakultesi, Isparta, Turkey tel: +90 5323468258,fax: +90 2462324510,e-mail:drercanvarol@yahoo.com 